\title{
Proposing a Flowchart to Detect English Functional Equivalents for Persian Lexis: A Practical Approach
}

\author{
Alireza Bahremand \\ Faculty of English Language and Literature, College of Literature and Humanities, Velayat University, Iranshahr, Iran \\ Mousa Mahmoudzehi \\ Faculty of Persian Literature, College of Literature and Humanities, Velayat University, Iranshahr, Iran
}

\begin{abstract}
To translator trainers, teaching translation from trainees' native to their foreign or second language is far more demanding than the other way round especially when it comes to detecting functional equivalence for the lexis of the source language. On the other hand, reaching a formulaic roadmap to English functional equivalence for Persian lexes would contribute to machine translation technology inasmuch as it can form the backbone of Persian-to-English digital applications and software. The present study aims at detecting a schematic approach to assist Persian-to-English translation trainees as well as translation software developers in detecting functional equivalents for Persian lexes. To this end, based upon the information gathered through observing an authentic translation process by a professional translator and following an interview with him in order to elucidate unclear points, a short, clear and to-the-point algorithm flowchart made up of two terminals, four decision diamonds and three process boxes was devised. Moreover, different sources such as dictionaries, thesauri, lexicons, and so forth used by the translator in question during the process of translation were introduced, along with a discussion of where, when and how they ought to be used.
\end{abstract}

Index Terms - flowchart, functional equivalent, Persian lexis, professional translator, translation software, translation training

\section{INTRODUCTION}

Translation has long been considered a linguistic activity (Way, 2016). Through the process of translation, detecting lexical dynamic equivalence is so delicate a task that demands much tactfulness in visiting minor differences in the sense of lexical items, both in terms of linguistics and pragmatics. However, the challenge would be even more serious if translation is to be made from translators' mother language to their foreign or second language as, apart from linguistic and pragmatic propriety, naturalness of the translation product would gain salience. Also how to translate the localism into authentic English has become a focus of public attention and aroused a heated discussion in the translation circle (Yang-Yan, 2016). Accordingly, there seems to be a roadmap needed for not only Iranian translator trainees, but also computer programmers to yield user-friendly, clear and to-the-point algorithms which would lead to English lexical dynamic equivalents for Persian terms. Such algorithm, besides acquainting translator trainees with a real-world example of how professional translators tackle the problems related to finding foreign language functional equivalents vis-a-vis their mother-tongue lexes, would constitute the backbone of computer-assisted translation software; thus, paving the way for both pedagogical and technological objectives. Besides Translators' individual traits are responsible for their different behavior and hence their success or failure in the translation process. It means translators' traits (i.e., their personality enduring characteristics) affect their decision making process, which leads to dissimilar performances of different translators and, above all, their success or failure in different translation situations, such as translation of various texts (Shaki-Khoshsaligheh , 2017).

\section{A. Equivalence and Equivalence Effect}

It would be possible to trace the roots and origins of the paradigm of "equivalence" back to the times of ancient Greece, the Roman Empire and figures such as Cicero, but going through all stages and epochs of history in which the principles of "equivalence" had appeared in some form or another, would go beyond ( Lottes, 2018). Playing crucial roles in the process of translation, equivalence and equivalent effect constitute fundamental concepts in translation studies. While in formal equivalence the focus of attention is upon the form and the content of the message, dynamic (functional) equivalence is based on the principle of equivalent effect, that is, when the substantial relation between the translation and its readers is basically the same as the relation between the original work and its receptors in the source language (Nida, 1964). Meanwhile, it is assumed that the knowledge and ability in equivalents are indicatives of translators' competence in translation (Koller, 1979). Koller also differentiated five types of equivalent relations: denotative, connotative, text-normative, pragmatic and formal equivalence (Koller, 1979). From among Koller's five 
types of equivalence, pragmatic equivalence is similar to Nida's dynamic equivalence (Munday, 2013). Hence, what Nida (1964) called dynamic or functional, and Koller (1979) named pragmatic equivalence should be the minimum criteria to be met by translators in order to make their translation communicatively acceptable.

\section{B. Linguistic Competence and Performance}

In generative transformational grammar, linguistic competence refers to the whole knowledge that a person has acquired or learned from the grammar of a given language. It also includes their ability to comprehend and produce sentences of the language which they have never heard before (Chomsky, 1957). Nowadays linguistic competence is one of the most demanded. It guarantees technical university graduates the possibility of global employment, on the one hand, and the chance of getting cutting edge education in leading training centers of the world, on the other hand (Chistyakova et al 2017). On the other hand, linguistic performance refers to the actual application of language in their speech or writing (Chomsky, 1957). However, one's linguistic performance could not always be a true milestone to determine the extent of their competence, because the latter might have been affected by physiological or psychological intrusions such as the context of the utterance, fatigue or the emotional status of the utterer (Fromkin et al 2018). Since the realm of the native language competence of an individual is naturally more extended than that of their second language, the Iranian translation trainees, while translating from Persian to English, need to be far more tactful in choosing target language lexical items than the time when they translate the other way around. To put it another way, Iranian students translating from English to Persian can enjoy the fairly trustable gauge of their mother tongue linguistic competence whereas such instrument is not normally at hand when they translate from Persian to English. That should prove the necessity of a road map to guide them on the path to reach lexical dynamic equivalence through English language. Therefore we can say that no progress can be facilitated without translation. Considering the importance of translation in our daily life, and the role of conveying meaning in this regard, it has received scant attention from teachers and learners (Khavari-Ahmadian, 2018).

\section{Lexical Adjustment}

Mollanazar (2008) regarded lexical adjustment as change of form. He classified lexical adjustment into two categories of redistribution of semantic components and change in part of speech before subcategorizing the first category into "analytical" and "synthetic" processes (Mollanazar, 2008). He considered a process through which a single word in the source language expands to make up a phrase or clause in the target language as "analytical", and the process of reduction of several components in the source language into one item in the target language as a "synthetic" process of redistribution of syntactic components (Mollanazar, 2008). He also considered change in part of speech as the second approach to lexical adjustment (Mollanazar, 2008). However, while assuming the process of change in part of speech as "transposition", Vinay and Darbelnet (1995) considered it as a structural change in the process of translation. The fact that lexical adjustment is more or less intertwined with structural adjustment doubles the necessity of a strategy to apply with much tact in choosing lexical equivalents on part of the translator during the process of translation. Beside lexical adjustment there should be also grammatical adjustment which is a kind of changing form. It is the process of adjusting grammatical pattern from source language into target language (Afifah et al 2018).

\section{Word Formation Processes}

It is believed that vocabulary knowledge had a significant relationship with translation performance. However, this relationship could not contribute significantly to the translation performance (Kafipour- Jahansooz, 2017). When it comes to lexical equivalence, a number of processes are used by linguists to describe how different words are formed. Coinage (invention), compounding, blending, clipping (truncation), backformation, conversion, acronyms, derivation, echoism (symbolism), onomatopoeia, folk etymology and reduplication are among word-formation processes assumed by linguists to account for the advent of words into languages (Yule, 2016). Multiple processing could also be applied to form neologisms. Hence, a student seeking to come up with lexical functional equivalence has to choose among different ways to employ the right diction that is the very fact which makes their task even more challenging.

\section{E. Delicacies in the Sense of Words}

There is no clear-cut meaning associated with words, but rather there are several layers of meaning communicated even though a term is uttered within the same context. Thus far, several ways have been proposed by linguists to categorize meaning. Leech (1980) classified meaning as: conceptual, connotative, stylistic, affective, reflected, collocational and topical. Leech (1980) clarified that conceptual meaning is organized mainly through distinctive features. For example, the words woman and boy are comparable through features: HUMAN, MALE and ADULT:

Woman $=+$ HUMAN, - MALE, + ADULT

Boy $=+$ HUMAN, + MALE, -ADULT

Leech's conceptual meaning is more or less corresponding to the referential meaning proposed by Halliday (1961) should the syntagmatic and paradigmatic axes of De Saussure (1916) be applied to it (Lotfipour-Saedi, 2001). He further maintained that connotative meaning, rather than referring to the mere denotative content of a term, refers to the communicative value of that. He clarified that besides indicating the physical features (+ HUMAN, - MALE, + ADULT), the term "woman" connotes tenderness, making a good cook, wearing dress, and so forth. Lotfipour-Saedi 
(2001) elaborated that the degree of formality of the context determines the stylistic meaning of a word. For example, steed, horse, nag and gee-gee enjoy the stylistic features of very poetic, neutral, colloquial and childish respectively. According to Lotfipour-Saedi (2001), should the range of linguistic context be further extended, the affections of the utterer will be reflected in the affective meaning.

One of the different conceptual meanings of a word might assume dominance owing to its more familiarity, frequency of use, or capability of mental association on part of the receiver of the utterance. Information that involves prior knowledge is more easily remembered than completely new information and People benefit from integration of the newly acquired information with pre-existing knowledge within the relevant area (Ning X etal., 2018). LotfipourSaedi (2001) exemplified the Comforter and the Holy Ghost as referring to the same concept, whereas the former inspires a desirably sweet effect, but the latter a feeling of terror. He explained that the collocated meaning of a term is determined by its neighboring words or phrases as far as that adjacency would sound natural (Lotfipour-Saedi, 2001). The attributive adjectives pretty and handsome, while sharing the same conceptual meaning of good-looking, precede different nouns inasmuch as the former collocates with female human beings and the latter with male ones.

As for translation trainees, it seems essential that, while deciding to choose the closest lexical equivalent for a given term, they see into different layers of meaning to spot semantic delicacies which could be achieved through checking different sources and references.

In order to fulfill the above-mentioned aspects of necessity for a clear-cut, strict and user-friendly roadmap to English functional equivalents for Persian lexes available to translator trainees and translation software developers, the present study seeks to answer the following research questions:

1. As a professional translator, what process does Mokhtari Ardekani (2018) practically undergo to come up with English functional equivalents for Persian lexes?

2. Based upon the practical process undergone by Mokhtari Ardekani (2018), what schematic model may Iranian translation trainees or equivalent software developers adopt to come up with English lexical equivalence for Persian Lexis?

\section{METHODS}

The present study employs both descriptive and prescriptive approaches to research inasmuch as the descriptive part as Williams and Chesterman (2014) asserted deals with "workplace studies" as a subcategory of studies on "translation process". Williams and Chesterman (2014) further explained that such an approach is to observe a given translator or translators during a defined period in their everyday work, perhaps prior to interviews. Based on this view and upon securing his consent, Mokhtari Ardekani was chosen as the professional translator to be observed during translating Shazde-Ehtejab, a work by the Iranian novelist Golshiri (2000) because the novel abounds in culture-specific terms which needs meticulous tact in detecting functional equivalents thereof. The aforementioned translator was chosen to observe due to the fact that apart from being available to the researcher, Mokhtari Ardekani is an experienced university professor, a lexicographer, and an author of 25 books, among which are books on both theories and practice of translation.

The methodology of the descriptive part of the survey is consisted of two parts. During the first part, Mokhtari Ardekani was observed for his translation procedures to be meticulously spotted and jotted down while no interruption was made on part of the researcher in order to keep the everyday natural atmosphere intact so that the translator would feel as if nobody were around to avoid any effects on either the process or the product of translation.

In the second phase, after the process of translation was completed, the translator was inquired for justifications for each of his decisions, so that the very reasons behind the measures taken by him during the process of translation could be elucidated.

Meanwhile, the methodology of the present research is not confined to a descriptive approach, as it also enjoys a prescriptive nature. According to Wollman (2018), a prescriptive or normative study is an applied rather than a theoretical approach trying to come up with an assertion, a solution or a proposal to address a known problem space. With the prescriptive side of the present study, the points acquired during the observation of Mokhtari Ardekani's authentic translation process were applied to the standard format of algorithm flowchart developing system to form different action symbols such as flow lines, terminals, processes, decisions, and so forth which are used in computer science to provide a schematic view of a given procedure.

Such an approach besides making a user-friendly roadmap for translator trainees, could be adopted to form the backbone of digital translation applications and software which are to be used as computer aided translation (CAT) tools.

Williams and Chesterman (2014) in defining translation with commentary as an area in translation research asserted that this commentary (or annotated translation), which is a form of introspective and retrospective research, will include some discussion of the translation assignment, an analysis of the aspects of the source text, and a reasoned justification of the kinds of solutions you arrived at for particular kinds of translation problem. The very recently mentioned function of annotated translation proposed by Williams and Chesterman (2014) strongly supports the idea that prescriptive methods are appropriate to be applied to translation studies. Besides the prescriptive approach to annotated translation, 
observing and post-observing supplementary interviews with a professional translator constitute the double-faceted theoretical framework of the present study.

\section{RESULTS}

Once translators find themselves wary of missing the equivalent of a Persian word or term in their mental corpora, an uphill struggle would start on their part to detect one which, apart from collocating with the co-text and context involved, could meet the pragmatic, psychological, sociological, cultural and mythological requirements of the text in order to contribute to the dynamism, functionality and naturalness of translation.

The aim of the present study is to identify a process flowchart that would start with the translators' awareness about the absence of an English functional equivalent of a term in Persian source text, and will end in such an equivalent at hand. To this end, upon monitoring a competent translator during his authentic process of translation, the standard format of flowchart development employed in computer sciences was applied to come up with different symbols such as flow lines, terminals, processes, decisions, and so forth; hence, as the usual format for all algorithms, the flowchart begins with a start terminal as in Figure 1:

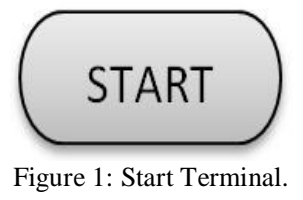

At the outset of the descriptive part of the study, Mokhtari Ardekani was observed trying to come up with functional equivalents for Persian lexes. Meanwhile, he was noted in contemplating as he came across with every Persian terms requiring English functional equivalents for translation. However, the profundity and length of contemplation varied with different cases. As mentioned in the methodology section above, the reason for such behavior of the translator was not to be enquired on the spot in order to keep the translation atmosphere as intact and natural as possible. Yet, through the post-translation interview, when asked for the reason(s) behind the given behavior, the translator answered that he was searching his mind for an English equivalent for the Persian word in question (Author, 2018). Thus, the process practically starts with Decision Diamond 1 (See Figure 2).

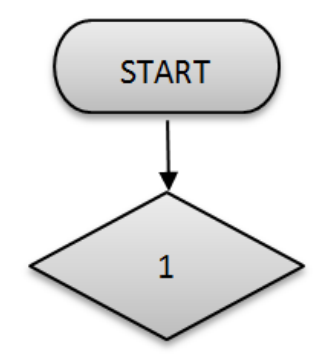

Figure 2: Decision Diamond 1 reads: “Does any tentative English equivalent exist in the translator's mind?”.

The three basic requirements for a person to be a translator are: proficiency in both source and target text and familiarity with the subject of the text to be translated. Therefore, a person who wishes to start translation task must make sure that, at the time of deciding to communicate either a concrete or an abstract concept, they can recall at least one term as its signifier. Mokhtari Ardekani further explained that a number of mind training exercises such as doing crossword puzzles can contribute to the formation of behavioral abilities of translator trainees to enable them to improvise English formal equivalents on the spot (Author, 2018).

The answer to Decision Diamond 1 which asks the translator whether any tentative English equivalent exists in their mind is logically either "yes" or "no" (See Figure 3).

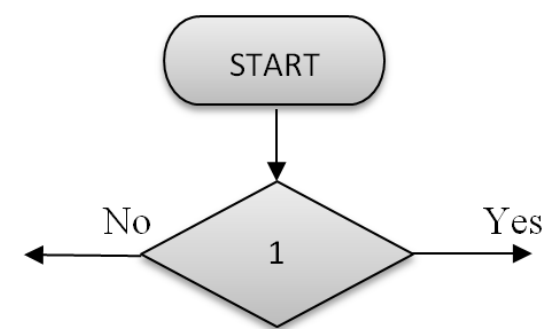

Figure 3: Decision Diamond 1 reads: “Does any tentative English equivalent exist in the translator's mind?". 
Provided that the answer be positive, the flowchart would go on through the right-side flow-line to Decision Diamond 2. (See Figure 4).

The next behavior of Mokhtari Ardekani after his intellectual strife to detect a tentative English equivalent was the frequent checking of mono-lingual dictionaries. During the post-translation interview, when asked for the reason of such behavior, Mokhtari Ardekani acknowledged that he wanted to ascertain that the term he had subjectively detected was the appropriate one (Author, 2018).

He further commented that even if a corresponding term is triggered as soon as the translator comes across with the source language word, they could never take it for granted since the term has yet to undergo different test plants and be examined against different touchstones (Author, 2018).

Accordingly, the first stage to be taken by the translator is checking the given term in a valid monolingual (in this case an English-to-English) dictionary.

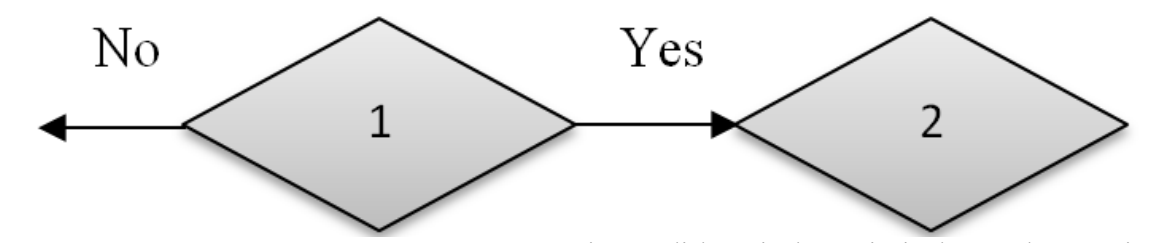

Figure 4: Decision Diamond 1 reads: "Does any tentative English equivalent exist in the translator's mind?"

Decision Diamond 2 reads: "According to monolingual dictionaries, is your choice semantically the right one?".

In fairly rare cases, the observed translator was detected referring to Persian-English dictionaries. When asked for the reason behind this very behavior, Mokhtari Ardekani asserted that he could think of no proper equivalents for the given term (Author, 2018).

Putting Mokhtari Ardekani's remarks in flowchart terms, however, if no English equivalent flashes in the mind of the translator spontaneously or after some interval of contemplating, the answer to Decision Diamond 1 would be "No". In this case the next step through the algorithm would be Process Box 1 (See Figure 5).

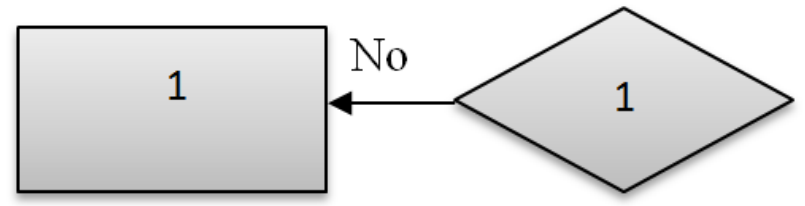

Figure 5: Decision Diamond 1 reads: "Does any tentative English equivalent exist in the translator's mind?".

Process Box 1 reads: "Choose your tentative English equivalent from a good Persian-to-English dictionary."

Through his post-translation interview, Mokhtari Ardekani further explained that after consulting trustworthy Persian-to-English dictionaries and picking up the lexical item which is deemed to be the most suitable, it should be double-checked through a monolingual dictionary (Author, 2018).

Accordingly, the flowchart will continue to look as Figure 6.

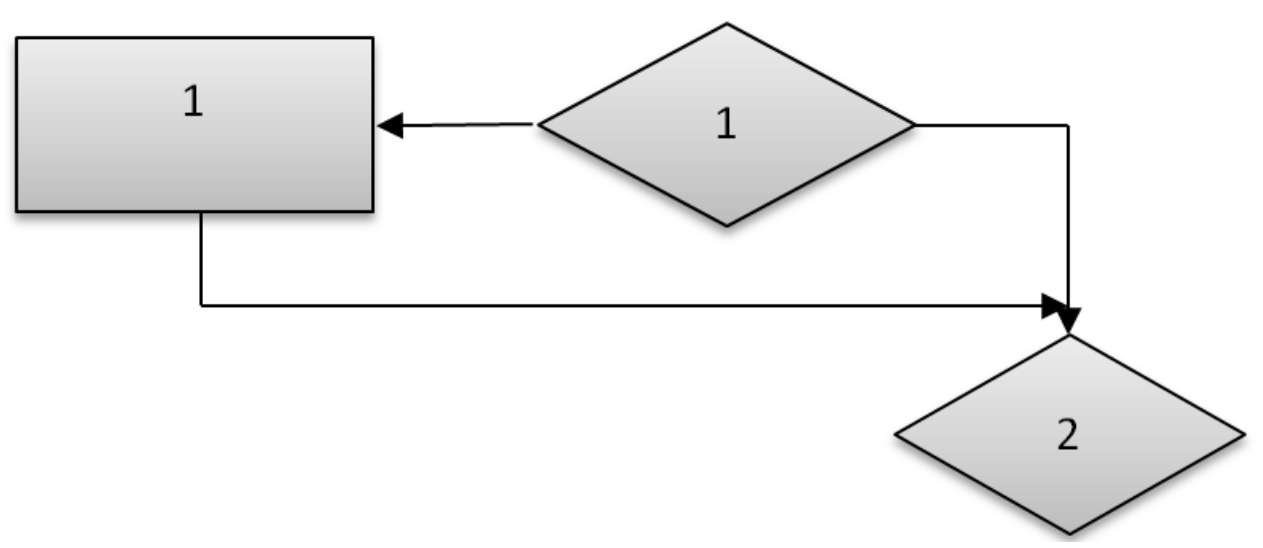

Figure 6: Decision Diamond 1 reads: "Does any tentative English equivalent exist in the translator's mind?". Decision Diamond 2 reads: "According to monolingual dictionaries, is your choice semantically the right one?". Process Box 1 reads: "Choose your tentative English equivalent from a good Persian-to-English dictionary."

Through the next step, Mokhtari Ardekani was monitored referring to the Longman Lexicon of Contemporary English. After the translation, when asked for the reason, he maintained that when the translator has established that the chosen lexical equivalent is verified by a trustable monolingual dictionary, the next step is to decide its appropriateness as far as pragmatics, psychology, sociology, culture, mythology, and so forth are concerned. Mokhtari Ardekani 
contended that the most upright way in doing so is to refer to sources and references such as Longman Lexicon of Contemporary English (Author, 2018).

McArthur and McArthur (1981) stipulated that the Lexicon provides the translator with the sense of words through fourteen "semantic fields" of a pragmatic and everyday nature. They further maintained that such references, in addition to telling apart the subtle differences among the meanings of the words, which seem to be synonymous at the first glance, elucidate the pragmatic delicacies of each term through providing perspicuous examples typed in italics as well as pictorial illustrations and figures in order to add to the intelligibility of exemplifications (McArthur \& McArthur, 1981). Hence, upon referring to the Lexicon, the translator would be able to determine the most suitable choice not only in terms of linguistics, but also pragmatics, psychology, sociology, culture, mythology, and so forth; thus, further approaching a more dynamic and functional equivalent.

Accordingly, the proposed flowchart will continue from Decision Diamond 2 to Decision Diamond 3 (See Figure 7).

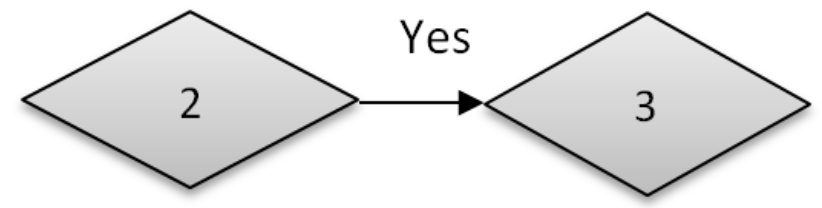

Figure 7: Decision Diamond 2 reads: “According to monolingual dictionaries, is your choice semantically the right one?".

Decision Diamond 3 reads: "Does the Lexicon verify your choice in terms of pragmatics, psychology, sociology, culture, mythology, etc.?"

Upon using the Longman Lexicon of Contemporary English, Mokhtari Ardekani was observed checking reference books known as thesauri. Mokhtari Ardekani later justified his doing so by stipulating that if according to monolingual dictionaries, the term chosen to fill the gap of the right lexical functional equivalent is not the proper one, the translator must refer to a thesaurus (Author, 2018).

Thesauri (the plural form of thesaurus) are references which provide terms with a list of synonyms and antonyms. Through the interview, Mokhtari Arkani suggested that translators, through using thesaurus, choose a synonym for the word refuted through checking the Lexicon (Author, 2018).

With respect to Mokhtari Ardekani's suggestion, the no-flow line of Decision Diamond 2 would lead to the Process Box 2 (Figure 8).

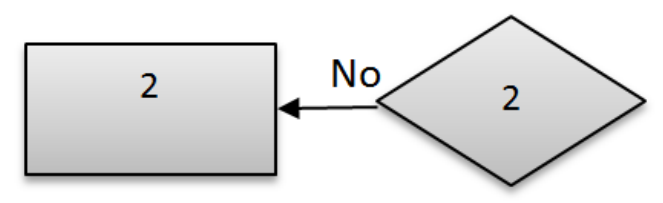

Figure 8: Decision Diamond 2 reads: "According to monolingual dictionaries, is your choice semantically the right one?". Process Box 2 reads: "Choose a synonym in a dictionary of synonyms and antonyms (Thesaurus)."

The translator trainee must use the refuted word by Decision Diamond 2 as an entry to check the thesaurus. Upon deciding over a synonym derived from using the refuted word applied to Decision Diamond 2 as the entry of the thesaurus, the flowchart will loop to the same to check whether the new term is the right semantic equivalent in accordance with monolingual dictionaries (See Figure 9).

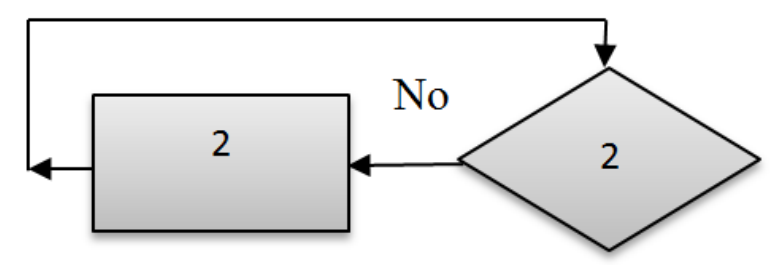

Figure 9: Decision Diamond 2 reads: “According to monolingual dictionaries, is your choice semantically the right one?". Process Box 2 reads: "Choose a synonym in a dictionary of synonyms and antonyms (Thesaurus)."

Provided that Decision Diamond 3 which checks references like Lexicon verifies the choice as the right functional equivalent in terms of pragmatics, sociology, culture, mythology and so forth, the next step would be checking for collocation. In this respect, Mokhtari Ardekani used dictionaries of collocation. According to McIntosh, Francis, and Poole (2009), "Collocation is the way words combine in a language to produce natural sounding speech and writing". They clarified that although both strong and heavy, for example, are adjectives to intensify nouns, strong wind and heavy rain constitute more natural (native-like) compounds than when they modify rain and wind respectively 
(McIntosh et al 2009). Mokhtari Ardekani asserted that translator trainees can consult a number of dictionaries such as Oxford Collocations Dictionary for Students of English to come up with natural-sounding combinations, namely collocations (Author, 2018).

Hence, the flowchart would go on to Decision Diamond 4. (See Figure 10)

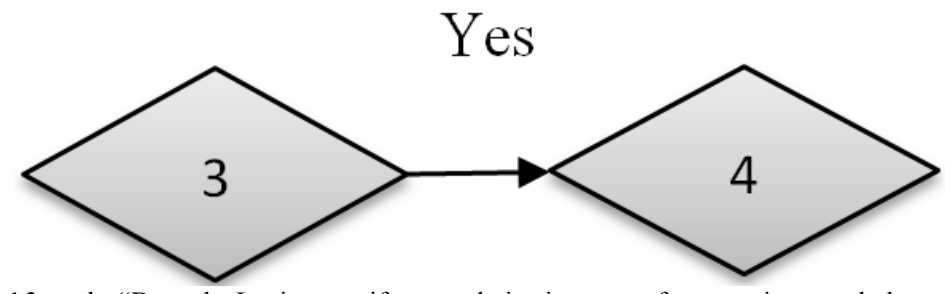

Figure 10: Decision Diamond 3 reads: "Does the Lexicon verify your choice in terms of pragmatics, psychology, sociology, culture, mythology, etc.?".

Decision Diamond 4 reads: "According to dictionaries of collocation, does your choice collocate with neighboring terms?".

Still, it was seen in some cases that upon referring to the Lexicon, the translator in question would return to the Thesaurus. Mokhtari Ardekani later accounted for such an attitude by asserting that he had learned through the Lexicon that his provisional term was not the right one in terms of psychology, sociology, culture, mythology, and so forth (Author, 2018). That was the reason why, for the second time, he referred to the Lexicon to choose a synonym from the list provided therein.

Translating Mokhtari Ardekani's behavior to flowchart algorithm language, the process flowchart would return to Process Box 2 in case the answer to Decision Diamond 3 is negative. Accordingly, the algorithm will continue to look like Figure:11.

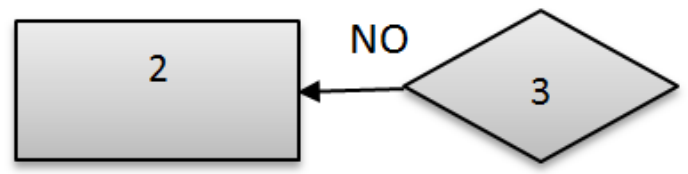

Figure 11: Decision Diamond 3 reads: "Does the Lexicon verify your choice in terms of pragmatics, psychology, sociology, culture, mythology, etc.?".

Process Box 2 reads: "Choose a synonym in a dictionary of synonyms and antonyms (Thesaurus)."

Provided that the tentative term to be used as the lexical functional equivalent was approved by the Oxford Collocations Dictionary for Students of English to collocate with its neighboring words, Mokhtari Ardekani made his mind to use it as his final choice (Author, 2018). Hence, should the term collocate with its co-texts, the translator is allowed to use it in their translation product which would be the last process box in the algorithm; thus, reaching the END terminal (See Figure 12).

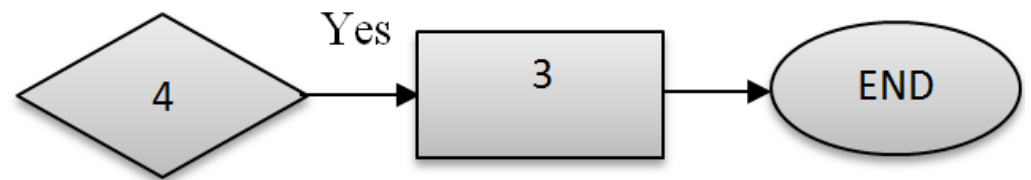

Figure 12: Decision Diamond 4 reads: "According to dictionaries of collocation, does your choice collocate with neighboring terms?". Process Box 3 reads: "Use the term."

However, it was seen occasionally that, after checking the collocation of a word, Mokhtari Ardekani would return to the thesaurus. In his post-translation interview, Mokhtari Ardekani emphasized that if the given word does not collocate with the co-texts, the translator had better modify the neighboring words to collocate with the given term instead (Author, 2018). Should it be impossible to do so, the final resort will be referring to the Thesaurus once more to choose another synonym (Author, 2018).

Thereupon, in such case, there will be a further loop from Decision Diamond 4 to Process Box 2 through which the translator must consult the Thesaurus to reconsider a synonym which would go through Decision Diamonds 1, 2 and 3 (See Figure 13). 


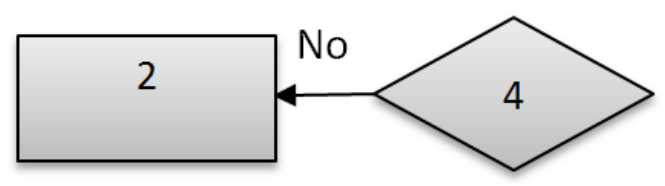

Figure 13: Decision Diamond 4 reads: "According to dictionaries of collocation, does your choice collocate with neighboring terms?". Process Box 2 reads: "Choose a synonym in a dictionary of synonyms and antonyms (Thesaurus)."

\section{DISCUSSION AND LIMITATIONS}

Meticulous inspection of Mokhtari Ardekani's translation process as well as the post-translation interview with him paved the way for would-be-translators who wish to be provided with a strict, clear and to-the-point path, toward coming up with English functional equivalents for Persian non-technical lexes. The devised algorithm flowchart could also form the backbone of CAT (Computer Assisted Translation) tools, applications and software which are intended for the same purpose.

Moreover, 13 distinct figures, each indicating one single stage of the whole process, were introduced. Still, to achieve the goal stated in the research question 2, an integrated whole of the process is required. Thus the integration of the thirteen formerly discussed algorithm commands is manifested through Figure 14:

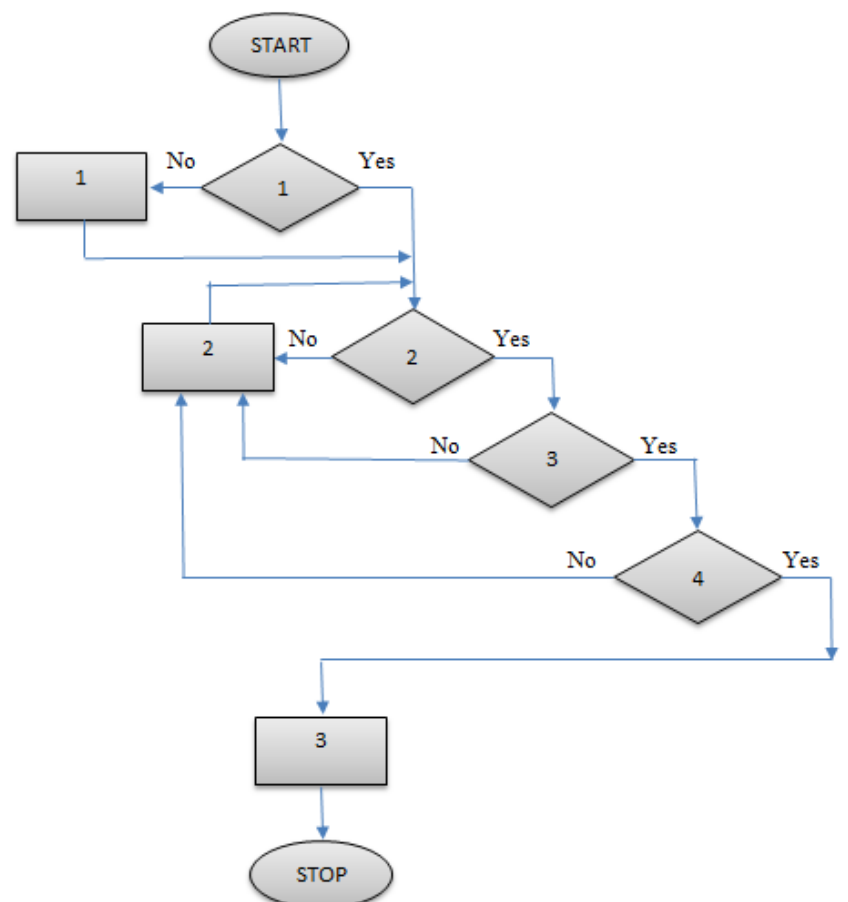

Figure 14: Decision Diamond 1 reads: "Does any tentative English equivalent exist in the translator's mind?".

Decision Diamond 2 reads: "According to monolingual dictionaries, is your choice semantically the right one?"

Decision Diamond 3 reads: "Does the Lexicon verify your choice in terms of pragmatics, psychology, sociology, culture, mythology, etc.?".

Decision Diamond 4 reads: "According to dictionaries of collocation, does your choice collocate with neighboring terms?".

Process Box 1 reads: "Choose your tentative English equivalent from a good Persian-to-English dictionary."

Process Box 2 reads: "Choose a synonym in a dictionary of synonyms and antonyms (Thesaurus)."

Process Box 3 reads: "Use the term."

Applying the whole procedures practiced by Mokhtari Ardekani during detecting English functional equivalents for Persian lexes to algorithm flowchart developing system, it was concluded that such a process starts with Decision Diamond 1. Should the translator assume any terms as the equivalent, the answer would be positive and they have to go on to Decision Diamond 2. However, in case there exists no equivalents in the translator's intuition to be tentatively chosen, the answer to Decision Diamond 1 will be negative. In this case, the next step will be going on to Process Box 1 which is referring to Persian-to-English dictionaries. To Mokhtari Ardekani and many other scholars and professional translators, although there are presently many newly-published copies of Persian-to-English dictionaries available in the market, Haim (1993) should prove to be the best despite the fact that it was first published more than half a century ago (Author, 2018). Mokhtari Ardekani elaborated that Kimia Persian-English Dictionary by Emami (2006) is the next reliable source to be used by translator trainees at this stage. Yet, the latter is not so comprehensive as the former one is (Author, 2018). 
Once the translator has undergone Process Box 1, the next step would be the same as if there were an equivalent in their mind; thus, according to Decision Diamond 2, they should check the same through a valid monolingual dictionary to make sure that their choice is semantically right.

This way, they can ascertain that the equivalent in question, besides bearing the intended meaning, has not been inspired into the translator's mind owing to linguistic flaw or spoonerism. The more proficient the translator within the target language, the more capable they could be in filling lexical gaps they encounter during translation process. According to Mokhtari Ardekani, however, translators ought not to rely solely on the equivalent which they deem as proper, but they have to double-check their linguistic competence by looking the term up in a valid monolingual dictionary (Author, 2018).

Furthermore, Mokhtari Ardekani warned that looking up the mere definition of the word would not suffice. Rather, the translator might as well check the examples provided after each definition, the part(s) of speech, the usage label(s), and even the right spelling(s) provided by English monolingual dictionaries (Author, 2018). In addition, while checking the part of speech, the translators are recommended not to restrict themselves to the main items such as verb, noun, adjective or adverb, but also look for more specific categories, that is to see whether the given noun is countable or uncountable, the adjective involved is predicative or attributive, or the verb in question is a regular or an irregular one (Author, 2018). Seeing into the verb patterns will also add to the naturalness of the translated text. More importantly, translators must check the usage label of the word they intend to use (Author, 2018). Mokhtari Ardekani further reminded translators that the usage label of a word sheds light on the style or the register of the context which should embed the word (Author, 2018). According to Yorkey (1970), the style of a term determines the degree of its formality which should accord with the context in which it appears. A usage label indicating formal, informal, colloquial, slang, obscene or taboo would reveal the style of the term (Yorkey, 1970). Wardhaugh (2011) defined registers as sets of vocabulary items associated with different occupational or social groups. Yorkey (1970) exemplified surgeons, airline pilots, bank managers, sales clerks, jazz fans who use different vocabularies. Yorkey (1970) recommended using dictionaries that have been recently published or revised, by dissuading using dictionaries which are more than ten or at most fifteen years old. He warned about using pocket-sized dictionaries since they are abridgments of abridgments and copied without changes from older dictionaries (Yorkey, 1970). Mokhtari Ardekani contended that both paper and digital dictionaries released by Oxford, Longman, Webster's, Collins, Random House and American Heritage publications can be advised to the students at the first stage of coming up with lexical functional equivalence (Author, 2018).

The next step in passing through the algorithm starts with the positive answer to Decision Diamond 2. Should it be the case, the algorithm will undergo Decision Diamond 3. Yet, as long as reliable monolingual dictionaries do not approve the tentative equivalent which had formerly been chosen either by the translator or by a Persian-to-English dictionary, the algorithm would reach Process Box 2 which commands checking the term in question through thesauri. According to Kirkpatrick and Kirkpatrick (1994), thesauri are of two types: Roget-type and synonym-type. They maintained that while Roget-like thesauri are arranged according to theme, the headwords of the synonym-type thesauri are arranged in alphabetical order (Kirkpatrick \& Kirkpatrick, 1994). Mokhtari Ardekani asserted that upon deciding over a synonym in thesaurus, the chosen word must be checked against the standards of semantics through an English monolingual dictionary (Author, 2018). Accordingly, in flowchart terms, there would be a loop to Decision Diamond 2.

Through the next step, if the answer to Decision Diamond 3 is positive, that is the delicacies of meaning among synonymous words are discovered and the closest equivalent to the source language term is determined, the algorithm would reach Decision Diamond 4 which serves to the naturalness of speech by checking the given term through dictionaries of collocation. Still, provided that the answer to Decision Diamond 3 is not positive, and since the Lexicon would not approve the given term pragmatically, the second loop in the flowchart will start with Decision Diamond 3 and end in Process Box 2, which implies that a synonym must be chosen from the thesaurus.

According to Mokhtari Ardekani, the last step in the process of detecting functional equivalent for Persian lexis is to check the term verified by the Lexicon through a reliable dictionary of collocations (Author, 2018), that is Decision Diamond 4. In case the answer to the recent question is negative, the translation product will lack naturalness and sound awkward which in turn would diminish the stylistic elegance thereof. Should it be the case, the algorithm flowchart will face the second loop to Process Box 2 through which the thesaurus should help the translator to decide over another synonym to the word failing to collocate with co-texts. On the other hand, provided that the term collocates with neighboring words, the answer to Decision Diamond 4 will be positive, and according to the Process Box 4 , the translator can apply the term in their translation product; thus, ending the algorithm flowchart.

\section{CONCLUSION}

Translation is not a simple technical, but a socio-cultural, subjective and ideological process. In contrast to the translation turn in other disciplines, however, most qualitative and critical accounting research neglects translation as a methodological and epistemological (Evans, 2018). The results of the present study as well as the analysis provided through the discussion section would yield the answers to the research questions. As for research question 1 which inquires about the process which Mokhtari Ardekani (2018) practically undergoes as a professional translator to come up with English functional equivalents for Persian lexes, the study reveals that Mokhtari Ardekani 
(2018) makes use of a number of different sources and references such as monolingual and bilingual dictionaries, thesauri, dictionaries of collocations, and the Lexicon not haphazardly, but in a quite hierarchically rational order. On the other hand, the answer to the second research question which deals with the make-up of the schematic model to be adopted by Iranian translation trainees or equivalent software developers was the process model derived from the systematic study of Mokhtari Ardekani (2018) during his genuine process of detecting English functional equivalents for Persian lexes. Such a flowchart model was proven to consist of four decision diamonds, three process boxes and two terminals which are interrelated through twelve flow lines.

In brief, it is concluded that for the first part, the observed professional translator uses many source references in a quite rational order. Secondly, his procedure can be translated into a brief, clear and to-the-point algorithm flowchart to be adopted by both translator trainees and translation software developers.

Conducting research through observing professional translators while performing their routine task would yield invaluable facts from ups and downs of a genuine translation process. Upon doing so and through a didactic approach toward their study, researchers would find themselves as media between professional translators and the translator trainees who wish to acquire the knacks of translation not only theoretically, but also according to what a skillful and experienced translator practices in real life.

Further research can be conducted on proposing flowcharts which would come up with functional equivalents for technical terms (jargons), although the process seems not to be so demanding as it is for detecting functional equivalents for non-technical lexes. Moreover, developing algorithm flowcharts for detecting functional equivalents for grammatical units is much worthy of investigation since, once put into practice, it can reduce the syntactic flaw often abundantly spotted through the final products of Persian-to-English digital translation software and applications.

\section{REFERENCES}

[1] Afifah, L.N., Hartono, R., and Yuliasri, I. (2018). Translation technique and grammatical equivalence in Indonesian English translation of central java visitor guide. English Education Journal, 8(2), 254-264.

[2] Author, A. (2018). Post-translation Interview with Mokhtari Ardekani on the reasons behind his manner of translation. Paper presented at the the two-day meeting on linguistics of House of Culture, Kerman.

[3] Chistyakova, G., Bondareva, E., Demidenko, K., Podgornaya. E, and Kadnikova, O. (2017). Using innovative interactive technologies for forming linguistic competence in global mining education. E3S Web of conferences, 15, 04010.

[4] Chomsky, N. (1957). Syntactic structures. The Hague: Mouton.

[5] De Saussure, F. (1916). Cours de linguistique generale. Paris: Payot.

[6] Emami, K. (Ed.) (2006) Kimia Persian_English Dictionary, Tehran: Farhang Moaser.

[7] Fromkin, V., Rodman, R., and Hyams, N. (2018). An introduction to language, Fort Worth: Cengage Learning.

[8] Golshiri, H. (2000). Shazdeh Ehtejab. Tehran: Niloofar.

[9] Haim, S. (Ed.) (1993). Persian to English Dictionary, New York City: Hippocrene Books.

[10] Halliday, M. A. K. (1961). Categories of the theory of grammar. Word, 17(3), 44-59.

[11] Kafipour, R., and Jahansooz, N. (2017). Effect of content schema, vocabulary knowledge and reading comprehension on translation performance. Indonesian EFL Journal: Journal of ELT, Linguistics, and Literature, 3(10), 22-39.

[12] Khavari, S., and Ahmadian, M. (2018). An investigation on the relationship between the grammatical competence of young Iranian English translation stusents and their ability to translate from English to Farsi. SSYJ, 8(28), 45-58.

[13] Kirkpatrick, B., and Kirkpatrick, E. M. (1994). The Oxford paperback thesaurus: Oxford University Press London.

[14] Koller, W. (1979). Equivalence in translation theory (A. Chesterman, Trans.). In A. Chesterman (Ed.), Readings in translation theory. Helsinki: Oy Finn Lectura Ab. 99-104.

[15] Leech, G. (1980). Language and tact (Pragmatics and beyond series): Amsterdam: John Benjamins.

[16] Lotfipour-Saedi, K. (2001). An introduction to principles of translation: A discoursal approach. Tehran: Academic Publication Center.

[17] Lottes, D. (n.d.). What is equivalence - a concept explained by the pre-stage of translation studies. Retrieved June 4, 2018 from https://www.academia.edu/5665488/ Equivalence_in_the_Pre-Stage_of_Translation_Studies.

[18] McArthur, T., and McArthur, T. G. (1981). Longman lexicon of contemporary English: Longman London.

[19] McIntosh, C., Francis, B., and Poole, R. (2009). Oxford Collocations Dictionary for Students of English: A Corpus-Based Dictionary with CD-ROM Which Shows the Most Frequently Used Word Combinations in British and American English: Oxford: Oxford University Press.

[20] Mokhtari Ardekani, M. A. (2018). Shazdeh Ehtejab. Translation. In Press.

[21] Mollanazar, H. (2008). Principles and methodology of translation (10 ed.). Tehran: SAMT.

[22] Munday, J. (2013). Introducing translation studies: Theories and applications. London, New York Routledge.

[23] Nida, E. A. (1964). Toward a science of translating: with special reference to principles and procedures involved in Bible translating: Brill Archive.

[24] Ning, X., Li, C., and Yang, J. (2018). Word Familiarity Modulated the Effects of Category Familiarity on Memory Performance. Frontiers in Psychology, 9, 1429. doi: 10.3389/fpsyg.2018.01429.

[25] Shaki, R., and Khoshsaligheh, M. (2017). Personality type and translation performance of Persian translator trainees. Indonesian Journal of Applied Linguistics, 7(2), 360-370.

[26] Vinay, J-P., and Darbelnet, J. (1995). Comparative stylistics of French and English: A methodology for translation. Amsterdam, Philladeiphia: John Benjamins Publishing. 11.

[27] Wardhaugh, R. (2011). An introduction to sociolinguistics. Malden: John Wiley \& Sons. 28. 
[28] Way, C. (2016). The challenges and opportunities of legal translation and translator training in the 21th century. International journal of communication, 10, 1009-1029.

[29] Williams, J., and Chesterman, A. (2014). The map: a beginner's guide to doing research in translation studies. London: Routledge.

[30] Wollman,

L. F. (2018).

Research

paradigms.

Retrieved

from https://www.chds.us/coursefiles/research/lectures/research_paradigms/script.pdf website on 11.12. 2018.

[31] Yang, M., and Yan, D. (2016). The translation strategies for Chinese diplomatic neologisms from the perspective of political equivalence. Babel, 62(4), 661-675.

[32] Yorkey, R. C. (1970). Study skills for students of English as second language. New York: McGraw-Hill.

[33] Yule, G. (2016). The study of language. Cambridge: Cambridge University Press.

Alireza Bahremand was born in Kerman, Iran in 1977. He received his BA degree in English translation from Shahid Bahonar University of Kerman in 2006 and his MA in the same field from Isfahan University in 2009.

$\mathrm{He}$ is currently a member of the Faculty of English Language and Literature at Velayat University, Iranshahr, Iran where he has been TEACHING and DOING RESEARCH on theory and practice of translation in addition to TRANSLATING and PREPARING BOOKS AND MATERIALS for translator trainees for the last eleven years.

Mr Bahremand's research interests include translation training, history of translation and interpretation on which he has published nine articles and books so far.

Mousa Mahmoudzahi was born in Iranshahr, Iran in 1960. He has a Ph.D. in studies of ancient cultures and languages from Azad University, Tehran. He is currently an associate professor of the Faculty of Persian Literature at Velayat University, Iranshahr where he has been TEACHING and DOING RESEARCH for nearly twenty-five years. His areas of research include old Iranian cultures and languages with a focus on Balochi, historical linguistics and etymology on which he has published 25 articles and books so far.

Dr. Mamoudzehi is a member of The Linguistic Society of Iran (LSI) as well as the Editorial Board of Zabanshenakht Journal. 